\title{
Approximation and Inaproximability Results on Balanced Connected Partitions of Graphs
}

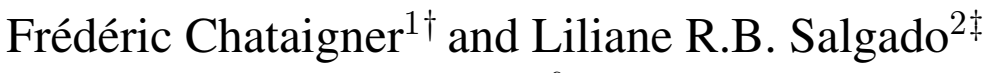 \\ and Yoshiko Wakabayashi非 \\ ${ }^{1}$ Instituto de Matemática e Estatística, Universidade de São Paulo, Rua do Matão 1010 - Cidade Universitária - CEP \\ 05508-090, São Paulo, SP, Brazil. Email: fred@ime.usp.br, yw@ime.usp.br \\ ${ }^{2}$ Centro de Informática, Universidade Federal de Pernambuco, Av. Prof. Luis Freire, s/n-Cidade Universitária - \\ CEP 50732-970 - Recife, PE, Brazil. Email: Iiliane@cin. ufpe.br
}

received March 31, 2007, revised June 29, 2007, accepted August 21, 2007.

Let $G=(V, E)$ be a connected graph with a weight function $w: V \rightarrow \mathbb{Z}_{+}$and let $q \geq 2$ be a positive integer. For $X \subseteq V$, let $w(X)$ denote the sum of the weights of the vertices in $X$. We consider the following problem on $G$ : find a $q$-partition $P=\left(V_{1}, V_{2}, \ldots, V_{q}\right)$ of $V$ such that $G\left[V_{i}\right]$ is connected $(1 \leq i \leq q)$ and $P$ maximizes $\min \left\{w\left(V_{i}\right): 1 \leq i \leq q\right\}$. This problem is called Max Balanced Connected q-Partition and is denoted by $\mathrm{BCP}_{q}$. We show that for $q \geq 2$ the problem $\mathrm{BCP}_{q}$ is NP-hard in the strong sense, even on $q$-connected graphs, and therefore does not admit a FPTAS, unless $\mathrm{P}=\mathrm{NP}$. We also show another inapproximability result for $\mathrm{BCP}_{2}$. For the problem $\mathrm{BCP}_{q}$ restricted to $q$-connected graphs, it is known that for $q=2$ the best result is a $\frac{4}{3}$-approximation algorithm obtained by Chlebíková; for $q=3$ and $q=4$ we present 2-approximation algorithms. When $q$ is not fixed (it is part of the instance), the corresponding problem is called Max Balanced Connected Partition, and denoted as BCP. We show that BCP does not admit an approximation algorithm with ratio smaller than $6 / 5$, unless $\mathrm{P}=\mathrm{NP}$.

Keywords: spproximation algorithm, balanced connected partition, hardness of approximation, PTAS

\section{Introduction}

There are many applications in image processing, data bases, operating systems and cluster analysis [2, 5, 14, 15, 17] that can be modelled as a problem of breaking a connected graph into a certain number of "balanced" connected subgraphs. Given an input graph $G=(V, E)$ with weights on the vertices, we may formalize the concept of "balancedness" as follows: find a partition of the vertex set $V$ into $q$ classes $V_{1}, V_{2}, \ldots, V_{q}$ such that the weight of the 'lightest' class is as large as possible. The requirement that makes the problem interesting, and also difficult, is that each class has to induce a connected subgraph of $G$. This is the problem that we focus in this paper, called here Max Balanced Connected q-Partition

\footnotetext{
${ }^{\dagger}$ Supported by FAPESP, Proc. 05/53840-0 (postdoctoral fellowship); originally from France.

¥Supported by FAPESP, Proc. 98/02407-9 (PhD scholarship).

$\S$ Supported by CNPq (Proc. 490333/04-4 and 308138/04-0) and PRONEX-FAPESP/CNPq (Proc. 03/09925-5).
} 
Problem $\left(\mathrm{BCP}_{q}\right)$, and defined formally in Section 2 We also consider a variant of this problem, denoted as BCP, in which $q$ (the number of desired classes) is given as part of the instance.

The design of approximation algorithms and the study of approximability properties of NP-hard combinatorial optimization problems are topics of research that have received much attention in the last decade. The problem $\mathrm{BCP}_{q}$ has been investigated under this perspective, but the existing results are very incipient, and there is still much to be done to have a complete understanding of its approximability properties. This problem is also known as Max-min q-Partition Problem [3, 4, 20].

The simpler unweighted version of $\mathrm{BCP}_{q}$ is the special case of $\mathrm{BCP}_{q}$ in which all vertices have weight 1 . This version will be denoted by $1-\mathrm{BCP}_{q}$. We call attention of the reader to the fact that whenever we refer to the problems $\mathrm{BCP}_{q}$ or $1-\mathrm{BCP}_{q}$, unless otherwise stated, we are assuming that the input graph is simply connected. Sometimes we shall refer to these problems on graphs with higher connectivity or some other special property. We do not use a different notation in this case, but we stress that the problem is on (or for) such class of graphs. Thus, we may refer to $\mathrm{BCP}_{q}$ on $q$-connected graphs or $\mathrm{BCP}_{2}$ on 3 -connected graphs.

It is easy to prove that $1-\mathrm{BCP}_{2}$ is solvable in polynomial time for 2-connected graphs. It is also polynomial for graphs in which every block has at most two articulation points [1, 7, 9, 10], and graphs in which every block has at most $p$ articulation points connected as a clique [1], where $p$ is a constant. Dyer and Frieze [8] proved that for every $q \geq 2$ the problem $1-\mathrm{BCP}_{q}$ is NP-hard (even for bipartite graphs). When the input graph has a higher connectivity, then the following result for $1-\mathrm{BCP}_{q}$ has been proved by Lovász [13] (see also Györi [12]).

Theorem 1 (Lovász) Let $G$ be a q-connected graph with $n$ vertices, $q \geq 2$, and let $n_{1}, n_{2}, \ldots, n_{q}$ be positive natural numbers such that $n_{1}+n_{2}+\ldots+n_{q}=n$. Then $G$ has a connected q-partition $\left(V_{1}, V_{2}, \ldots, V_{q}\right)$ such that $\left|V_{i}\right|=n_{i}$ for $i=1,2, \ldots, q$.

Efficient algorithms for 1-BCP ${ }_{q}$ on $q$-connected graphs appeared in the nineties. Initially, for the cases $q=2$ and $q=3$ [23, 24]. Later, polynomial algorithms for all $q \geq 2$ were also obtained [16]. In 1977, Nakano, Rahman and Nishizeki [18] designed a linear time algorithm to find connected 4-partitions of 4-connected planar graphs [18].

For the more general weighted case, it is known that $\mathrm{BCP}_{q}$ is polynomially solvable only for ladders [4] and for trees [20]. Becker, Lari, Lucertini and Simeone [3] showed that $\mathrm{BCP}_{q}$ restricted to grids $G_{m \times n}$, with $n \geq 3$ is already NP-hard. They also designed approximation algorithms for which estimates for the (relative) error are given under certain conditions; general approximation ratios are not given, except for $q=2$, for which a $3 / 2$-approximation can be guaranteed.

For the problem $\mathrm{BCP}_{2}$ the following results have been proved: it is NP-hard on connected graphs [6], bipartite graphs [8], and graphs with at least one block containing $\Omega(\log |V|)$ articulation points [1]. In the case of complete graphs, although apparently simpler, it is still NP-hard (a result easy to be proved). In terms of algorithms, the best result is a $\frac{4}{3}$-approximation algorithm obtained by Chlebíková [7] in 1996. It is not known whether $\mathrm{BCP}_{q}$ admits a PTAS.

The remaining of this paper is organized as follows. In Section 2 we give some definitions and establish the notation. In Section 3 we prove that for every $q \geq 2$ the problem $\mathrm{BCP}_{q}$ is NP-hard in the strong sense even for $q$-connected graphs. We also show some inapproximability results: we prove that there is no $(1+\epsilon)$-approximation algorithm for the problem $\mathrm{BCP}_{2}$, where $\epsilon \leq 1 / n^{2}$ and $n$ is the number of vertices of the input graph, unless $\mathrm{P}=\mathrm{NP}$. We also prove that $\mathrm{BCP}$ does not admit a PTAS; more precisely, we prove that $\mathrm{BCP}$ does not admit an $\alpha$-approximation algorithm with $\alpha<6 / 5$, unless $\mathrm{P}=\mathrm{NP}$. In Section 4 
we present a 2-approximation algorithm for $\mathrm{BCP}_{3}$ on 3-connected graphs. In Section 5 we generalize the ideas of the previous section and present a 2-approximation for $\mathrm{BCP}_{4}$ on 4-connected graphs.

Some of the results we shall present here have appeared in [22]. This paper contains the proofs that were omitted and additional new results.

\section{Definitions and Notation}

Let $G=(V, E)$ be a connected graph with a weight function $w: V \rightarrow \mathbb{Z}_{+}$. For simplicity, we may also say that $G$ is a $w$-weighted graph. For any subset $V^{\prime} \subseteq V$, we denote by $G\left[V^{\prime}\right]$ the subgraph of $G$ induced by $V^{\prime}$; and we denote by $w\left(V^{\prime}\right)$ the sum of the weights of the vertices in $V^{\prime}$, that is, $w\left(V^{\prime}\right)=$ $\sum_{v \in V^{\prime}} w(v)$. Throughout this paper we assume that $q$ is a positive integer, $q \geq 2$. Given a connected graph $G=(V, E)$, a partition $\left(V_{1}, V_{2}, \ldots, V_{q}\right)$ of $V$ such that $G\left[V_{i}\right]$ is connected for $i=1,2, \ldots, q$ is called a connected q-partition. The Max Balanced Connected q-Partition Problem $\left(\mathrm{BCP}_{q}\right)$ consists in finding for a given $w$-weighted connected graph $G=(V, E)$, a connected $q$-partition $\left(V_{1}, V_{2}, \ldots, V_{q}\right)$ that maximizes the function $\min \left\{w\left(V_{i}\right): 1 \leq i \leq q\right\}$. We say that the value $\min \left\{w\left(V_{i}\right): 1 \leq i \leq q\right\}$ is the measure of the $q$-partition $\left(V_{1}, V_{2}, \ldots, V_{q}\right)$. The problem BCP is a slight variant of $\mathrm{BCP}_{q}$ : it differs only in the fact that the number $q$ of desired classes is given as part of the instance.

Let $P$ be an optimization problem and $\mathcal{A}$ an algorithm for $P$. For any instance $I$ of $P$, we denote by $\mathcal{A}(I)$ the solution returned by algorithm $\mathcal{A}$ for $I$, and opt $(I)$ the value of an optimal solution for $I$. Let $r: \mathbb{N} \rightarrow[1, \infty)$. If $P$ is a maximization problem, then we say that $\mathcal{A}$ is an $r(n)$-approximation algorithm if $\mathcal{A}$ runs in polynomial time, and, for any instance $I$ of $P$ of size $n$, the ratio $\operatorname{opt}(I) / \mathcal{A}(I)$ is at most $r(n)$. In this case, we say that $\mathcal{A}(I)$ is an $r(n)$-approximate solution for $I$. An algorithm $\mathcal{A}$ is a PTAS, Polynomial Time Approximation Scheme (resp. FPTAS, Fully Polynomial Time Approximation Scheme), if for any instance $I$ of $P$ and for any rational $\epsilon>0, \mathcal{A}$ returns a feasible solution $\mathcal{A}_{\epsilon}(I)$ such that the ratio $\operatorname{opt}(I) / \mathcal{A}_{\epsilon}(I)$ is at most $1+\epsilon$ in time bounded by a polynomial in $|I|$ (resp. polynomial both in $|I|$ and $1 / \epsilon)$.

\section{NP-completeness and hardness of approximation}

As the problem 1- $\mathrm{BCP}_{q}$ on $q$-connected graphs can be solved in polynomial time, a natural question is whether this also holds for the weighted version. We prove that this is not the case. We prove in this section that $\mathrm{BCP}_{q}$ restricted to $q$-connected graphs is NP-complete in the strong sense. We also show other results on the hardness of approximation of $\mathrm{BCP}_{2}$ and $\mathrm{BCP}$ (for arbitrary graphs).

We show first that the decision version of Max Balanced Connected 2-Partition Problem $\left(\mathrm{BCP}_{2}\right)$ is NP-complete in the strong sense for 2-connected graphs. In our proof we use similar ideas from that presented by Galbiati, Maffioli and Morzenti [6] for connected and unweighted graphs. We note that the result proved by Becker et al. [3] on the NP-completeness of $\mathrm{BCP}_{2}$ restricted to grids (and thus for 2 -connected graphs) is not in the strong sense.

For this purpose, consider the following NP-complete problem (see [19]), denoted by X3C, which is a variant of the Exact Cover by 3-Sets. Given a set $X$ with $|X|=3 q$ and a family $C$ of 3 -element subsets of $X,|C|=3 q$, where each element of $X$ appears in exactly 3 sets of $C$, decide whether $C$ contains an exact cover for $X$, that is, a subcollection $C^{\prime} \subseteq C$ such that each element of $X$ occurs in exactly one member of $C^{\prime}$.

Theorem 2 The decision version of $\mathrm{BCP}_{2}$ is NP-complete in the strong sense for 2-connected graphs. 
Proof: Given an instance $(X, C)$ of X3C, let $G=(V, E)$ be the graph with vertex set $V=X \cup C \cup\{a, b\}$ and edge set $E=\bigcup_{j=1}^{3 q}\left[\left\{C_{j} x_{i} \mid x_{i} \in C_{j}\right\} \cup\left\{C_{j} a\right\} \cup\left\{C_{j} b\right\}\right]$. Clearly, $G$ can be constructed in polynomial time in the size of $(X, C)$. It is also not difficult to see that $G$ is 2-connected (see Figure 1 ).

Define a weight function $w: V \rightarrow \mathbb{Z}_{+}$as follows: $w(a)=9 q^{2}+2 q ; w(b)=3 q ; w\left(x_{i}\right)=3 q$ for $i=1, \ldots, 3 q$; and $w\left(C_{j}\right)=1$ for $j=1, \ldots, 3 q$. Note that $w(V)=2\left(9 q^{2}+4 q\right)$. We shall prove that $C$ contains an exact cover for $X$ if and only if $G$ has a connected 2-partition $\left(V_{1}, V_{2}\right)$ such that $\min \left\{w\left(V_{1}\right), w\left(V_{2}\right)\right\} \geq W / 2$, where $W:=w(V)$.

Given an exact cover $C^{\prime}$, consider the connected 2-partition $\left(V_{1}, V_{2}\right)$ of $G$, where $V_{1}=\{a\} \cup\left\{C_{j}\right.$ : $\left.C_{j} \notin C^{\prime}\right\}$ and $V_{2}=\{b\} \cup\left\{C_{j}, x_{i}: C_{j} \in C^{\prime}\right.$ and $\left.x_{i} \in C_{j}\right\}$. Since $C^{\prime}$ consists of $q$ subsets, we have $w\left(V_{1}\right)=w(a)+3 q-q=9 q^{2}+4 q=W / 2$.

Conversely, let $\left(V_{1}, V_{2}\right)$ be a connected 2-partition of $G$ such that $\min \left\{w\left(V_{1}\right), w\left(V_{2}\right)\right\} \geq W / 2$. Then $\min \left\{w\left(V_{1}\right), w\left(V_{2}\right)\right\}=W / 2$. Note that $a$ and $b$ cannot belong to the same set $V_{i}$, because $w(a)+w(b)=$ $9 q^{2}+5 q>W / 2$. Suppose, without loss of generality, that $a \in V_{1}$ and $b \in V_{2}$. No vertex of $X$ is in $V_{1}$, otherwise $w\left(V_{1}\right) \geq w(a)+3 q>W / 2$. Therefore $V_{1}$ contains the vertex $a$ and some vertices of $C$. Since $w\left(V_{1}\right)=W / 2=9 q^{2}+4 q$, this implies that $V_{1}$ contains exactly $2 q$ vertices of $C$. Thus, $V_{2}$ has precisely $q$ vertices of $C$. Since these $q$ vertices are independent and the vertices in $X \cup\{b\}$ are also independent, it is easy to verify that these $q$ vertices of $C$ belonging to $V_{2}$ define an exact cover for $X$.

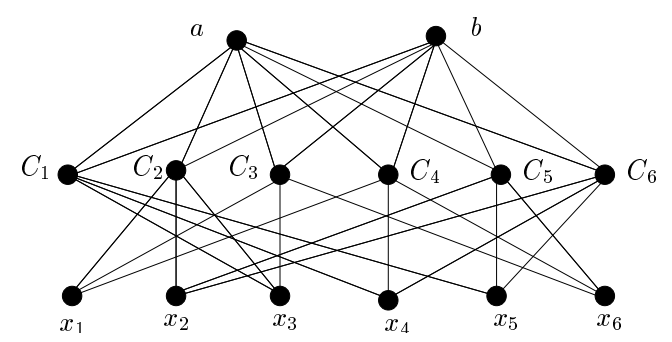

Fig. 1: Graph obtained by the reduction given in Theorem 2 for the instance $(X, C)$, where $C=\left\{C_{1}, C_{2}, \ldots, C_{6}\right\}$, $C_{1}=\left\{x_{3}, x_{4}, x_{5}\right\}, C_{2}=\left\{x_{1}, x_{2}, x_{3}\right\}, C_{3}=\left\{x_{1}, x_{3}, x_{6}\right\}, C_{4}=\left\{x_{1}, x_{4}, x_{6}\right\}, C_{5}=\left\{x_{2}, x_{5}, x_{6}\right\}$ and $C_{6}=$ $\left\{x_{2}, x_{4}, x_{5}\right\}$

We can generalize Theorem 2 as follows.

Theorem 3 For every $q \geq 2$, the decision version of $\mathrm{BCP}_{q}$ is $\mathrm{NP}$-complete in the strong sense for q-connected graphs.

Proof: Denote by $\mathrm{DBCP}_{q}$ the decision version of $\mathrm{BCP}_{q}$. Suppose $q \geq 3$. We prove, by induction on $q$, that the problem $\mathrm{DBCP}_{q-1}$ can be reduced to the problem $\mathrm{DBCP}_{q}$.

Let $I=(G, w, m)$ be an instance of $\mathrm{DBCP}_{q-1}$ that consists of a $(q-1)$-connected graph $G=(V, E)$, a function $w: V \rightarrow \mathbb{Z}_{+}$and a positive integer $m$. The goal is to decide whether this instance has a solution with measure at least $m$.

We construct an instance $I^{\prime}=\left(G^{\prime}, w^{\prime}, m\right)$ of $\mathrm{DBCP}_{q}$ that consists of a $q$-connected graph $G^{\prime}=$ $\left(V^{\prime}, E^{\prime}\right)$, with $V^{\prime}=V \cup\left\{v^{\prime}\right\}$, where $v^{\prime} \notin V$, and $E^{\prime}=E \cup\left\{v^{\prime} u: u \in V\right\}$, and a function $w^{\prime}$ on the 
vertices of $G^{\prime}$ such that: $w^{\prime}\left(v^{\prime}\right)=w(V) /(q-1)$ and $w^{\prime}(v)=w(v)$ for each $v$ in $V$. It is obvious that $G^{\prime}$ can be constructed in polynomial time in the size of $I$ and $G^{\prime}$ is $q$-connected.

We claim that the instance $I$ of $\mathrm{DBCP}_{q-1}$ has a connected $(q-1)$-partition with measure at least $m$ if only if the instance $I^{\prime}$ of $\mathrm{DBCP}_{q}$ has a connected $q$-partition with measure at least $m$. In fact, let $P=\left(X_{1}, \ldots, X_{q-1}\right)$ be a connected $(q-1)$-partition of $G$ with measure at least $m$. In this case, $\left(X_{1}, \ldots, X_{q-1},\left\{v^{\prime}\right\}\right)$ is a connected $q$-partition of $G^{\prime}$ with measure at least $m$.

Now, suppose that $P^{\prime}=\left(X_{1}^{\prime}, \ldots, X_{q}^{\prime}\right)$ is a connected $q$-partition of $G^{\prime}$ with measure $m^{\prime}$, where $m^{\prime} \geq$ $m$. Without loss of generality, suppose that $X_{q}^{\prime}$ contains $v^{\prime}$ and $w^{\prime}\left(X_{1}^{\prime}\right) \leq w^{\prime}\left(X_{i}^{\prime}\right)$ for $2 \leq i \leq q-1$. Since $w^{\prime}\left(X_{q}^{\prime}\right) \geq w^{\prime}\left(V^{\prime}\right) / q$, we have that $m^{\prime}=w^{\prime}\left(X_{1}^{\prime}\right)$. Let $R=X_{q}^{\prime} \backslash\left\{v^{\prime}\right\}$. If $R=\emptyset$, then $\left(X_{1}^{\prime}, \ldots, X_{q-1}^{\prime}\right)$ is a connected $(q-1)$-partition of $G$ with measure $m^{\prime}$. If $R \neq \emptyset$, as $G$ is $(q-1)$-connected, we can distribute the vertices of $R$ among the sets $X_{i}^{\prime}$ for $1 \leq i \leq q-1$ in such a way that the new sets $X_{i}^{\prime} \cup R_{i}$, where $\bigcup_{i=1}^{q-1} R_{i}=R$, induce connected subgraphs of $G$. In this case, $\left(X_{1}^{\prime} \cup R_{1}, \ldots, X_{q-1}^{\prime} \cup R_{q-1}\right)$ is a connected $(q-1)$-partition of $G$ with measure at least $m^{\prime}$. Since $m^{\prime} \geq m$, the proof of the claim is complete.

To conclude the proof note that for $q=2$ the statement corresponds to Theorem 2 . Now for $q \geq 3$, in view of the above reduction we can conclude, by induction, that the result follows.

From the above result we obtain immediately the following inapproximability result for $q$-connected graphs.

Corollary 1 For every $q \geq 2$, the problem $\mathrm{BCP}_{q}$ restricted to q-connected graphs does not admit a FPTAS, unless $\mathrm{P}=\mathrm{NP}$.

Moving now to an inapproximability result for $\mathrm{BCP}_{2}$, we mention first the following result obtained by Chlebíková [7].

Theorem 4 (Chlebíková) For any rational $\delta>0$, it is NP-hard to find in polynomial time a solution for $\mathrm{BCP}_{2}$ with an absolute error guarantee of $n^{1-\delta}$, where $n$ is the number of vertices of the input graph $G$.

We note that the approximation measure mentioned in the above result is the absolute error. This lead us to ask what happens if we consider the ratio bound measure. A result we obtained in this direction is the following.

Theorem 5 There is no $(1+\epsilon)$-approximation algorithm for the problem $\mathrm{BCP}_{2}$, where $\epsilon \leq 1 / n^{2}$ and $n$ is the number of vertices of the input graph, unless $\mathrm{P}=\mathrm{NP}$.

Proof: Suppose there exists an algorithm $\mathcal{A}$ for $\mathrm{BCP}_{2}$, on graphs with $n$ vertices that is an $(1+\epsilon)$ approximation, where $\epsilon \leq 1 / n^{2}$. We show that this algorithm can be used to solve the X3C problem, obtaining a contradiction, unless $\mathrm{P}=\mathrm{NP}$.

Let $(X, C)$ be an instance of the $\mathrm{X} 3 \mathrm{C}$ problem where $C=\left\{C_{1}, C_{2}, \ldots, C_{3 q}\right\}$ is a family of subsets of $X=\left\{x_{1}, x_{2}, \ldots, x_{3 q}\right\}$. Construct a graph $G=(V, E)$ as follows. Set $V=C \cup X \cup\{a, b\}$ and $E=\bigcup_{j=1}^{3 q}\left[\left\{C_{j} x_{i} \mid x_{i} \in C_{j}\right\} \cup\left\{C_{j} a\right\} \cup\left\{C_{j} b\right\}\right]$. Assign weights $w(v)$ to the vertices $v$ of $G$ as follows: $w(a)=6 q^{3}+q^{2} ; w(b)=2 q^{2} ; w\left(C_{j}\right)=q$ for $j=1, \ldots, 3 q$; and $w\left(x_{i}\right)=2 q^{2}$ for $i=1, \ldots, 3 q$. Observe that $w(V)=2\left(6 q^{3}+3 q^{2}\right)$ and $|V|=6 q+2$.

Recall that the measure of a connected 2-partition $\left(V_{1}, V_{2}\right)$ of $G$ is $\min \left\{w\left(V_{1}\right), w\left(V_{2}\right)\right\}$. 
Claim 1 If there exists a subfamily $C^{\prime}$ of $C$ that covers $X$ exactly, then $G$ has a connected 2-partition with measure $w(V) / 2$.

Proof: Given an exact cover $C^{\prime}$ of $X$, consider the connected 2-partition $\left(V_{1}, V_{2}\right)$ of $G$, where $V_{1}=\{a\} \cup\left(C \backslash C^{\prime}\right)$ and $V_{2}=\{b\} \cup X \cup C^{\prime}$. Clearly, we have that $w\left(V_{1}\right)=6 q^{3}+3 q^{2}=$ $w(V) / 2=w\left(V_{2}\right)$.

Claim 2 If $G$ has a connected 2-partition $\left(V_{1}, V_{2}\right)$ with measure at least $w(V) / 2-q+1$, then there exists a subfamily of $C$ that covers $X$ exactly.

Proof: Let $\left(V_{1}, V_{2}\right)$ be a connected 2-partition of $G$ with measure $m$, such that $m \geq w(V) / 2-q+1$. Observe that $a$ and $b$ cannot belong to the same set $V_{i}(i=1,2)$. Otherwise, $a, b$ and $C_{j}$ for some $j \in\{1,2, \ldots, 3 q\}$ would belong to the same set and thus the weight of this set would be at least $6 q^{3}+3 q^{2}+q=w(V) / 2+q$. But, then $m \leq w(V) / 2-q$, a contradiction.

Suppose that $a \in V_{1}$ and $b \in V_{2}$. Using a similar counting argument, we can prove that $X \subseteq V_{2}$. As the subgraph induced by $V_{2}$ has to be connected, we have $\left|C \cap V_{2}\right| \geq q$. But $\left|C \cap V_{2}\right|>q$ implies that $\left|C \cap V_{1}\right| \leq 2 q-1$. In this case, $w\left(V_{1}\right) \leq 6 q^{3}+q^{2}+(2 q-1) q=6 q^{3}+3 q^{2}-q=w(V) / 2-q$, and we have a contradiction again. Thus $\left|C \cap V_{2}\right|=q$, and hence $C \cap V_{2}$ covers $X$ exactly.

Now let us show that, using the algorithm $\mathcal{A}$ (mentioned at the beginning of this proof), it is possible to solve the $\mathrm{X} 3 \mathrm{C}$ problem. Let $\mathcal{A}^{\prime}$ be the following algorithm for $\mathrm{X} 3 \mathrm{C}$. Given an instance $(X, C)$, construct a graph $G$, as described above. Now, apply the algorithm $\mathcal{A}$ to $G$. If this algorithm finds a connected 2-partition with measure at least $w(V) / 2-q+1$, then return the message " $C$ has an exact cover." If the connected 2-partition found has measure smaller than $w(V) / 2-q+1$, then return the message " $C$ does not have an exact cover."

Clearly, the algorithm $\mathcal{A}^{\prime}$ solves the $\mathrm{X} 3 \mathrm{C}$ problem. Indeed, suppose first that $C$ has an exact cover. Then, by Claim 1. $G$ has a connected 2-partition with measure $w(V) / 2$. Since the algorithm $\mathcal{A}$ is a $(1+\epsilon)$-approximation, where $\epsilon \leq 1 / n^{2}$ and $n=|V|$, then $\mathcal{A}$ returns a solution with measure $m \geq$ $\left(n^{2} /\left(n^{2}+1\right)\right)$ opt. As opt $=w(V) / 2$, it follows that

$$
m \geq\left(\frac{n^{2}}{n^{2}+1}\right) \frac{w(V)}{2}=\left(1-\frac{1}{n^{2}+1}\right) \frac{w(V)}{2}=\frac{w(V)}{2}-\frac{w(V)}{2\left(n^{2}+1\right)} .
$$

Since $n=6 q+2$ and $w(V)=2\left(6 q^{3}+3 q^{2}\right)$, we have that $w(V) / 2\left(n^{2}+1\right)<q$. Thus, $m \geq$ $w(V) / 2-q+1$.

Suppose now that $C$ does not have an exact cover. Then, by Claim 2 the graph $G$ has only solutions with measure smaller than $w(V) / 2-q+1$. In this case, obviously the algorithm $\mathcal{A}$ returns a solution with measure smaller than $w(V) / 2-q+1$.

As the construction of $G$ can be done in polynomial time in the size of $(X, C)$, and the algorithm $\mathcal{A}$ is polynomial in the size of $G$, it follows that $\mathcal{A}^{\prime}$ is polynomial in the size of $(X, C)$. This concludes the proof of the theorem.

This is — to our knowledge — the strongest inapproximability result for $\mathrm{BCP}_{2}$.

All the results shown so far are on the problem $\mathrm{BCP}_{q}$. When $q$ is part of the instance, that is, for the problem $\mathrm{BCP}$, the following stronger inapproximability result can be obtained. 
Theorem 6 The problem $\mathrm{BCP}$ does not admit an $\alpha$-approximation algorithm with $\alpha<6 / 5$, unless $\mathrm{P}=\mathrm{NP}$.

Proof: We show a reduction from $\mathrm{X} 3 \mathrm{C}$ to $\mathrm{BCP}$, with the following property. Given an instance $I$ of $\mathrm{X} 3 \mathrm{C}$, we construct an instance $I^{\prime}=(G=(V, E), w, Q)$ of BCP such that: either $I^{\prime}$ has an optimal solution with measure $w(V) / Q$ if $I$ has an exact cover, or it has an optimal solution with measure $\frac{5}{6} w(V) / Q$, if $I$ does not have an exact cover.

Let $I=(X, C)$ be an instance of X3C, where $C=\left\{C_{1}, C_{2}, \ldots, C_{3 q}\right\}$ is a family of subsets of $X=$ $\left\{x_{1}, x_{2}, \ldots, x_{3 q}\right\}$. Let $\epsilon>0$ be a small number. Construct an instance $I^{\prime}=I^{\prime}(\epsilon)=(G=(V, E), w, Q)$ of BCP in the following way:

- Take $Q=10 q$.

- For each $x_{i}$ in $X$, let $H\left(x_{i}\right)$ be the graph with 16 vertices, we will call gadget, defined as follows (see Figure 2). It consists of 3 vertical paths of length 3 , say $P_{1}, P_{2}$ and $P_{3}$, all ending at a common vertex $x_{i}$, and internally vertex-disjoint. Each such a path $P_{j}$ starts at a vertex named $t_{i, i_{j}}$. The start vertices $t_{i, i_{1}}, t_{i, i_{2}}, t_{i, i_{3}}$ correspond to the 3 sets $C_{i_{1}}, C_{i_{2}}, C_{i_{3}}$ that contain $x_{i}$. These vertices will be referred as $t$-vertices. For each of the 3 possible choices of two paths (among $P_{1}, P_{2}$ and $P_{3}$ ), we attach two other new vertices, as follows. Let $P_{j}=\left(t_{i, i_{j}}, z_{i, j}, y_{i, j}, x_{i}\right)$, for $j=1,2,3$. Take two new vertices $l_{i, 1}$ and $r_{i, 1}$ and attach each of them to the vertices $y_{i, 1}$ and $y_{i, 2}$; take two other new vertices $l_{i, 2}$ and $r_{i, 2}$ and attach each of them to the vertices $z_{i, 2}$ and $y_{i, 3}$; take two other new vertices $l_{i, 3}$ and $r_{i, 3}$ and attach each of them to the vertices $z_{i, 1}$ and $z_{i, 3}$.

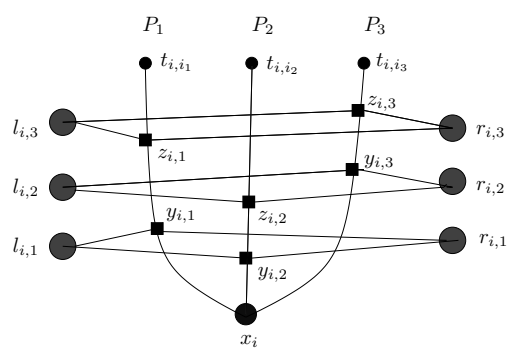

Fig. 2: The gadget $H\left(x_{i}\right)$

- Let $G=(V, E)$ be the graph obtained from the union of the gadgets $H\left(x_{i}\right), i=1, \ldots, 3 q$ with some additional $3 q$ vertices and $9 q$ edges, in the following way. Let $v_{1}, v_{2}, \ldots, v_{3 q}$ be the additional vertices, where each $v_{i}$ corresponds to a set $C_{i}$ of the instance $I$ of $\mathrm{X} 3 \mathrm{C}$. Now, whenever there is a set $C_{p}=\left\{x_{i}, x_{j}, x_{k}\right\}$ in the instance $I$, add three edges linking vertex $v_{p}$ to the vertices $t_{i, p}$, $t_{j, p}$ and $t_{k, p}$ of the gadgets $H\left(x_{i}\right), H\left(x_{j}\right)$, and $H\left(x_{k}\right)$, respectively. The vertices $v_{j}$ will be called $v$-vertices. In Figure 3 we show the graph $G$ that we obtain for the instance of $\mathrm{X} 3 \mathrm{C}$ mentioned in Figure 1

- Let $n$ be the number of vertices of the graph $G$ (note that $n=51 q$ ), and let $a$ be an integer such that $a \geq n / \epsilon$. 
- The weight function $w: V \rightarrow \mathbb{Z}_{+}$is defined as follows. We assign weight $2 a$ to the vertices $x_{i}$; weight $3 a$ to the vertices $l_{i, j}$ and $r_{i, j}, i=1, \ldots, 3 q, j=1,2,3$; and weight 1 to the remaining vertices. Note that each gadget has weight $20 a+9$ and $w(V)=(60 a+30) q$.

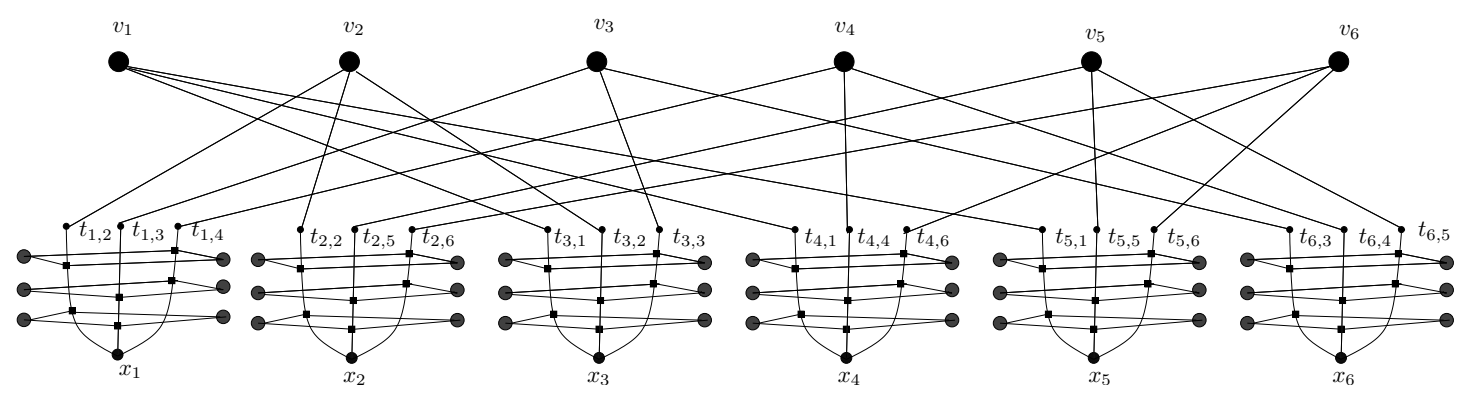

Fig. 3: The graph $G$ obtained for the instance of $\mathrm{X} 3 \mathrm{C}$ mentioned in Figure 1

In what follows, we refer to the connected subgraphs induced by a connected partition of the graph $G$ as the connected classes.

The idea behind the gadget $H\left(x_{i}\right)$ is the following: if the instance $I$ of $\mathrm{X} 3 \mathrm{C}$ has an exact cover, we want to ensure that the instance $I^{\prime}$ of BCP will have an optimal connected $Q$-partition with measure close to $6 a$. Such an optimal connected partition will consist of $10 q$ connected classes: $q$ of them are "induced" by an exact cover for $I$, and the other $9 q$ connected classes are those containing precisely one pair of vertices of type $l_{i, j}$ and $r_{i, j}$ that belong to a same gadget. Note that, since $w(V)=(60 a+30) q$, the measure of an optimal connected $10 q$-partition will not exceed $6 a+3$.

The role of the gadgets is also to guarantee that, if $I$ does not have an exact cover, then an optimal connected $10 q$-partition for the instance $I^{\prime}$ will have a measure smaller than $5 a+O(1)$.

We now make more precise and prove the statements above about the instances $I$ and $I^{\prime}$.

Claim 3 If the instance I has an exact cover, then $I^{\prime}$ has a solution with measure $6 a+O(1)$.

Proof: Let $C_{i_{1}}, C_{i_{2}}, \ldots, C_{i_{q}}$ be an exact cover for $I$. Construct a connected $10 q$-partition for the instance $I^{\prime}$ as follows. First, construct $q$ connected classes by considering the $q$ sets in the exact cover. (For example, for the instance $I$ corresponding to the graph shown in Figure 3 , consider the exact cover consisting of $C_{3}$ and $C_{6}$.) Each of these connected classes consists of a vertex $v_{j}$ corresponding to a set $C_{i_{j}}$ together with the 3 edges leaving it, each of them extended (in a connected way) with the unique vertical path that starts at one of its extremes (a $t$-vertex). Clearly, each of these $q$ connected classes has weight $6 a+10$. Consider the graph $G^{\prime}$ obtained from $G$ after removing the vertices in the $q$ connected classes we have constructed so far. The other $9 q$ connected classes can be obtained from $G^{\prime}$ as follows: first, in the remaining part of each gadget $H\left(x_{i}\right)$, construct 3 sets of paths, each one linking pairs of vertices of type $l_{i, j}$ and $r_{i, j}$. Note that this is possible, as only the vertices of one vertical path in each gadget were removed. Now, put each of the remaining vertices (all of weight 1 ) in any of the $10 q$ connected classes constructed so far, so as to obtain a connected $10 q$-partition of $G$. Clearly, all the connected classes have weight at least $6 a+1$. 
Claim 4 If $I^{\prime}$ has a solution with measure at least $(5+\epsilon) a$, then I has an exact cover.

Proof: Let $\left(V_{1}, V_{2}, \ldots, V_{Q}\right)$ be a solution of $I^{\prime}$ with measure at least $(5+\epsilon) a$. Since $n \leq \epsilon a$, the connected classes in this solution all have to contain one or more vertices with weigth $2 a$ or $3 a$, and therefore the weight of any connected class $G\left[V_{j}\right]$ must satisfy $K_{j} a \leq w\left(V_{j}\right)<K_{j} a+\epsilon a$, for some integer $K_{j}$.

Since the average weight of a connected class is $6 a+3$, if there existed a connected class with weight at least $7 a$, then there would exist another connected class with weight at most $5 a+6$, and therefore smaller than $(5+\epsilon) a$, a contradiction to our hypothesis.

Thus, $w\left(V_{j}\right)<7 a$, and therefore $w\left(V_{j}\right)=6 a+o(a)$, for $j=1, \ldots, Q$. Thus each connected class must contain either 2 vertices with weight $3 a$ or 3 vertices with weight $2 a$.

Let $Y$ be a connected class containing $x_{i}$. Suppose $Y$ contains no $t$-vertex. Then $Y$ can additionally contain only vertices with weights 1 or $3 a$ in the gadget $H\left(x_{i}\right)$, and therefore it will not have weight $6 a+o(a)$. Thus, $Y$ must contain a $t$-vertex of the gadget $H\left(x_{i}\right)$. Since $Y$ has weight $6 a+o(a)$, it has to contain precisely 3 vertices with weight $2 a$ and some vertices of weight 1 . But the only way to connect 3 vertices with weight $2 a$ is passing through a $v$-vertex $v_{j}$. Since the $v$-vertices have degree 3 , one of the two cases may happen: (1) either $Y$ contains exactly one vertex $v_{j}$, or (2) $Y$ contains at least two $v$-vertices.

In case (2), $Y$ must contain two $t$-vertices belonging to a same gadget, and furthermore they must be connected by a path contained in this gadget. In this case, since no vertex with weight $3 a$ can be used in such a path, two vertical paths in this gadget must be used. But then, these vertical paths separate a pair of vertices with weight $3 a$, and therefore some connected class will have weight $3 a$, a contradiction. Thus, case (1) must occur, and in this case, $Y$ contains 3 vertices with weight $2 a$, precisely one vertical path in the corresponding 3 gadgets and one vertex $v_{j}$. Since each vertex $x_{i}$ belongs to a connected class with precisely one $v$-vertex, there are exactly $q$ connected classes that induce an exact cover for the instance $I$ of X3C. This completes the proof of Claim 4.

Suppose there exists a polynomial time algorithm $\mathcal{A}_{\epsilon}$ for the problem BCP with approximation ratio $r(\epsilon) \leq 6 /(5+\epsilon)$, for $0<\epsilon<1$. Take an instance $I$ of X3C and reduce it (in polynomial time) to an instance $I^{\prime}$ of BCP, as we have shown, and apply the algorithm $\mathcal{A}_{\epsilon}$ to $I^{\prime}$. If $I$ has an exact cover, then by Claim 3 the instance $I^{\prime}$ has a solution with measure at least $6 a$. Thus, the algorithm $\mathcal{A}_{\epsilon}$ will find a solution for $I^{\prime}$ with measure at least $6 a / r(\epsilon) \geq(5+\epsilon) a$. If $I$ has no exact cover, then by Claim 4 an optimal solution for $I^{\prime}$ has measure smaller than $(5+\epsilon) a$. Thus, $I$ has an exact cover if and only if the algorithm $\mathcal{A}_{\epsilon}$ produces a solution with measure at least $(5+\epsilon) a$. Since X3C is NP-complete, such an algorithm $\mathcal{A}_{\epsilon}$ with approximation ratio $r(\epsilon) \leq 6 /(5+\epsilon)$ may not exist, unless $\mathrm{P}=\mathrm{NP}$.

Corollary 2 The problem $\mathrm{BCP}$ does not admit a PTAS, unless $\mathrm{P}=\mathrm{NP}$.

\section{Max balanced connected 3-partition}

In this section we present a 2-approximation algorithm for $\mathrm{BCP}_{3}$ restricted to 3 -connected graphs. Before that, we present some results that are useful in the analysis of the algorithm we propose. 
Theorem 7 Let $I$ be an instance of $\mathrm{BCP}_{q}, q \geq 2$, that consists of a w-weighted 2-connected graph $G=(V, E)$ such that $\max \{w(v): v \in V\} \geq W / q$, where $W=w(V)$. Then the following holds:

(a) The instance I has an optimal solution $\left(V_{1}^{*}, \ldots, V_{q}^{*}\right)$ such that $V_{i}^{*}=\left\{v^{*}\right\}$ for some $i$, $1 \leq i \leq q$, where $v^{*}=\arg \max \{w(v): v \in V\}$.

(b) If $q \geq 3$ and $\mathrm{BCP}_{q-1}$ admits an $r$-approximation algorithm for some $r$, then there exists an r-approximate solution for the instance I of $\mathrm{BCP}_{q}$.

The algorithm $\mathcal{A}$ for $\mathrm{BCP}_{3}$ that we describe makes use of the following property: if $G$ is a 3-connected graph with at least 5 vertices, then $G$ has an edge $e$ such that $G / e$, the graph obtained from $G$ by contracting $e$, is 3 -connected. In this case, we say that the edge $e$ is contractible.

The subroutine Contract-edge, used in this algorithm receives a 3-connected weighted graph and returns a 3-connected weighted graph that results from the contraction of an edge. If $G$ is a $w$-weighted graph, after the contraction of an edge $u v$, the new vertex that results from the identification of the vertices $u$ and $v$ receives weight $w(u)+w(v)$; and the weights of the other vertices do not change.

Another subroutine that is used in the algorithm is called BalBicon $_{2}$; this routine is precisely the $\frac{4}{3}$ approximation algorithm for $\mathrm{BCP}_{2}$ designed by Chlebíková [7]. As we need a few properties that are more precise than those in [7], we will essentially rephrase the results to establish these properties. Before we describe $\mathrm{BalBicon}_{2}$, let us present a concept that is used in the algorithm.

Let $G$ be a 2-connected graph and $\left(V_{1}, V_{2}\right)$ a connected 2-partition of $G$. We say that a vertex $u$ of $V_{2}$ is admissible (for $\left.V_{1}\right)$ if $\left(V_{1} \cup\{u\}, V_{2} \backslash\{u\}\right)$ is also a connected 2-partition of $G$. It is not difficult to prove that if $\left|V_{2}\right| \geq 2$, then there are at least two distinct vertices $v^{\prime}$ and $v^{\prime \prime}$ in $V_{2}$ that are admissible (for $V_{1}$ ). The proof of this statement can be easily obtained by considering the block graph of $G\left[V_{2}\right]$, and observing that it is a tree.

The algorithm BalBicon ${ }_{2}$ for $\mathrm{BCP}_{2}$ on 2-connected graphs, obtained by Chlebíková, works as follows. Let $G=(V, E)$ be the input graph and $w: V \rightarrow \mathbb{Z}_{+}$. Let $\beta:=w(V) / 2$ and $v_{1}$ be a vertex of maximum weight. Set $V_{1}:=\left\{v_{1}\right\}, V_{2}:=V \backslash V_{1}$. While $w\left(V_{1}\right)<\beta$ perform the following steps: (a) find a vertex $u \in V_{2}$ that is admissible and has the minimum weight; (b) If $w(u)<2\left(\beta-w\left(V_{1}\right)\right)$ then update the partition: take $V_{1}:=V_{1} \cup\{u\}$ and $V_{2}:=V_{2} \backslash\{u\}$; else leave the while-loop. Return the partition $\left(V_{1}, V_{2}\right)$.

We now present the result of Chlebíková [7] in a parameterized form (hidden in the proof she gave), as we need it in the sequel.

Theorem 8 (Chlebíková) Let I be a instance of $B C P_{2}$ which consists of a 2-connected graph $G=(V, E)$ and a function $w: V \rightarrow \mathbb{Z}_{+}$. Let $V=\left\{v_{1}, v_{2}, \ldots, v_{n}\right\}, n \geq 3, w\left(v_{1}\right) \geq w\left(v_{2}\right) \geq \ldots \geq w\left(v_{n}\right)$ and $t:=w(V) / w\left(v_{3}\right)$. Then, the algorithm $\mathrm{BalBicon}_{2}$, applied to I returns in polynomial time a connected 2-partition of $G$ with measure $m$, such that

(1) If $w\left(v_{1}\right) \geq \frac{1}{2} w(V)$ then $m=\operatorname{opt}(I)$;

(2) If $w\left(v_{1}\right)<\frac{1}{2} w(V)$ then $m \geq \frac{1}{2}\left[w(V)-w\left(v_{3}\right)\right] \geq \frac{t-1}{2 t} w(V)$;

(3) $\frac{\operatorname{opt}(I)}{m} \leq \frac{2 t-4}{t-1}$, if $3 \leq t \leq 4$; and $\frac{\operatorname{opt}(I)}{m} \leq \frac{t}{t-1}$, if $t \geq 4$.

The next two results follows immediately from Theorem 8 and the fact that $t \geq 3$. 
Corollary 3 The algorithm BalBicon 2 is a 4/3-approximation for $\mathrm{BCP}_{2}$ restricted to 2-connected graphs. Moreover, the ratio $4 / 3$ is tight.

Corollary 4 Let $I$ be an instance of $\mathrm{BCP}_{2}$ which consists of a 2-connected w-weighted graph $G=$ $(V, E)$. Let $v_{1}$ be a vertex of $V$ such that $w\left(v_{1}\right)=\max \{w(v) \mid v \in V\}$. If $w\left(v_{1}\right) \leq w(V) / 2$, then the algorithm $\mathrm{BalBicon}_{2}$ applied to the instance I returns in polynomial time a connected 2-partition with measure at least $w(V) / 3$.

We note that the ratio $4 / 3$ of algorithm BalBicon $_{2}$ is tight. To see this, consider the 2 -connected graph $G=(V, E)$ exhibited in Figure 4 (the weights are indicated on the vertices). Note that $w(V)=24, \beta=$ 12 and $t=4$. If the algorithm BalBicon 2 chooses first the vertex $v_{1}$ then it outputs a connected 2-partition with measure $m=9=w\left(v_{1}\right)+w\left(v_{4}\right)+w\left(v_{5}\right)$. As opt $(I)=12$, we have that $\operatorname{opt}(I) / m=12 / 9=4 / 3$.

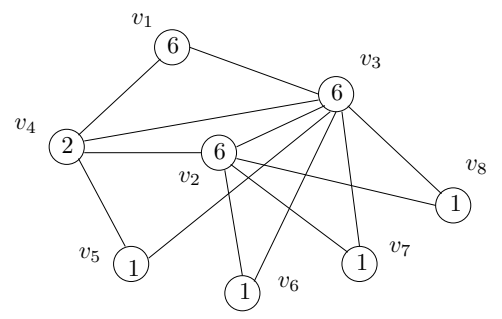

Fig. 4: An example showing that the ratio $4 / 3$ is tight

Now we are ready to describe the algorithm $\mathcal{A}$ for $\mathrm{BCP}_{3}$ on 3-connected graphs.

\section{Algorithm $\mathcal{A}_{3}$}

Input: A 3-connected graph $G=(V, E)$ and $w: V \rightarrow \mathbb{Z}_{+}$.

Output: A connected 3-partition of $G$.

1. $v_{1}=\arg \max \{w(v) \mid v \in V\}$.

2. $X=\left\{v_{1}\right\}$.

3. if $w(X) \geq w(V) / 3$ then

3.1. Let $G^{\prime}=G-X$ and $w^{\prime}$ be the restriction of $w$ to $G^{\prime}$.

3.2. $(Y, \bar{Y})=\operatorname{BalBicon}_{2}\left(G^{\prime}, w^{\prime}\right)$.

3.3. return $(X, Y, \bar{Y})$ and halt.

4. if $w(X)<w(V) / 6$ then

4.1. $(\widehat{G}, \widehat{w})=(G, w) ; \widehat{V}=V$.

4.2. while there is no vertex $\widehat{v} \in \widehat{V}$ such that $\widehat{w}(\widehat{v}) \geq \widehat{w}(\widehat{V}) / 6$ do

$(\widehat{G}, \widehat{w})=\operatorname{Contract}-\operatorname{edge}(\widehat{G}, \widehat{w})$.

4.3. $X=\{\widehat{v}\}$, where $\widehat{w}(\widehat{v}) \geq \widehat{w}(\widehat{V}) / 6$.

5. $G^{\prime}=\widehat{G}-X, w^{\prime}=\widehat{w}$.

6. $(Y, \bar{Y})=\operatorname{BalBicon}_{2}\left(G^{\prime}, w^{\prime}\right)$.

7. if $X \neq\left\{v_{1}\right\}$ then reconstruct from $(X, Y, \bar{Y})$ the 3-partition $(X, Y, \bar{Y})$ of $G$.

8. return $(X, Y, \bar{Y})$. 
The next result will be used to show the approximation ratio of algorithm $\mathcal{A}_{3}$.

Lemma 1 Let $G=(V, E)$ be a 3-connected graph and $w: V \rightarrow \mathbb{Z}_{+}$. Let $p$ be an integer, $p \geq 1$, $G_{1}=G$, and for $i=1, \ldots$, p let $G_{i+1}=G_{i} / e_{i}$, where $e_{i}$ is a contractible edge of $G_{i}$. Suppose that after the contraction of each edge $e=x y$, the weight of the new vertex $v_{e}$ is defined as $w\left(v_{e}\right)=w(x)+w(y)$, and the other vertices keep their weights. If $Q_{p}=\left(\tilde{V}_{1}, \ldots, \tilde{V}_{q}\right)$ is a connected q-partition of $G_{p}$, then this partition induces a connected q-partition $\left(V_{1}, \ldots, V_{q}\right)$ of $G$ such that $w\left(\tilde{V}_{i}\right)=w\left(V_{i}\right)$ for $i=1, \ldots, q$.

Proof: We show this result by induction on $p$. For $p=1$, consider $G_{1}=G / e_{1}$, and suppose that $e=e_{1}=x y$. Let $\left(\tilde{V}_{1}, \ldots, \tilde{V}_{q}\right)$ be a connected $q$-partition of $G_{1}$. Without loss of generality, suppose that $v_{e} \in \tilde{V}_{1}$. We can obtain a connected $q$-partition $\left(V_{1}, \ldots, V_{q}\right)$ of $G$ by taking $V_{i}=\tilde{V}_{i}$ for $i=2, \ldots, q$ and $V_{1}=\left(\tilde{V}_{1} \backslash\left\{v_{e}\right\}\right) \cup\{x, y\}$. Clearly this $q$-partition has the required properties.

Suppose that the statement holds when we contract up to $p-1$ edges, $p \geq 2$. Let us prove that it also holds when we perform $p$ contractions. Consider the graph $G_{p}=G_{p-1} / e_{p-1}$. By mimicking our proof for $p=1$, given a connected $q$-partition $Q_{p}=\left(\tilde{V}_{1}, \ldots, \tilde{V}_{q}\right)$ of $G_{p}$, we know how to construct a connected $q$-partition $Q_{p-1}$ of $G_{p-1}$. At this point, by our inductive hyphotesis, from $Q_{p-1}$ we can obtain a connected $q$-partition $Q=\left(V_{1}, \ldots, V_{q}\right)$ for the original graph $G$ such that $Q$ has the required properties.

Theorem 9 The algorithm $\mathcal{A}_{3}$ is a 2-approximation for $B C P_{3}$ restricted to 3-connected graphs.

Proof: Let $I=(G, w)$ be an instance of $\mathrm{BCP}_{3}$ that consists of a 3-connected graph $G=(V, E)$ and a function $w: V \rightarrow \mathbb{Z}_{+}$. Suppose that $v_{1}=\arg \max \{w(v) \mid v \in V\}$. Let $(X, Y, \bar{Y})$ be a solution returned by the algorithm $\mathcal{A}_{3}$ applied to the instance $I$ and let $m=\min \{w(X), w(Y), w(\bar{Y})\}$.

By Lemma 1 and Theorem 8 , we first note that the solution returned by $\mathcal{A}_{3}$ is a connected 3-partition. We also observe that, since the graph $\widehat{G}$ at the end of step 4.2 of algorithm $\mathcal{A}_{3}$ is 3 -connected, then the graph $G^{\prime}$ constructed in step 5 is 2-connected. Thus, the algorithm BalBicon ${ }_{2}$ is used apropriately in step 6. Note that the procedure Contract-edge can always be executed since at the moment of the call of this procedure the graph $\widehat{G}$ is 3 -connected and it has at least 6 vertices. By Lemma 1 , we also remark that the reconstruction mentioned in step 7 can be performed. It is not difficult to conclude that $\mathcal{A}_{3}$ can be implemented to run in polynomial time.

Next we analyse three cases to show its approximation ratio.

CASE 1 . There exists $v \in V$ such that $w(v) \geq w(V) / 3$.

In this case, we have that the 3-partition $(X, Y, \bar{Y})$ was returned in step 3.3. By Theorem 7 , the quality of the solution is that guaranteed by the algorithm BalBicon $_{2}$ used in step 3.2. As a consequence, by Corollary 3 we conclude that opt $(I) \leq(4 / 3) m$.

CASE 2. There exists $v \in V$ such that $w(V) / 6 \leq w(v)<w(V) / 3$.

In this case, $X=\left\{v_{1}\right\}$ and $w(V) / 6 \leq w(X)<w(V) / 3$. (a) If $m=w(X)$, then clearly, $m \geq$ $w(V) / 6$. (b) Assume $m=\min \{w(Y), w(\bar{Y})\}$. In this case, $(Y, \bar{Y})=\operatorname{BalBicon}_{2}\left(G^{\prime}, w^{\prime}\right)$, where $G^{\prime}=$ $\left(V^{\prime}, E^{\prime}\right)$ and $V^{\prime}=V \backslash X$. Hence, $w\left(V^{\prime}\right)>(2 / 3) w(V)$, since $w(X)<w(V) / 3$. As $w(v)<w(V) / 3$ for each $v \in V^{\prime}$, we have that $w(v)<w\left(V^{\prime}\right) / 2$. Thus, by Corollary 4 we have that $m \geq w\left(V^{\prime}\right) / 3$. Hence, $m>(2 / 9) w(V)$.

Thus, in both cases (a) and (b) we have that $m \geq w(V) / 6$. Since opt $(I) \leq w(V) / 3$, we obtain $\operatorname{opt}(I) \leq 2 m$. 
CASE 3. $w(v)<w(V) / 6$ for each $v \in V$.

By step 4.3 of the algorithm, we have that $X=\{\widehat{v}\}$, where $\widehat{w}(\widehat{v}) \geq \widehat{w}(\widehat{V}) / 6$. Let us consider two cases. (a) If $m=w(X)$, then $m \geq \widehat{w}(\widehat{V}) / 6=w(V) / 6$. (b) Assume $m=\min \{w(Y), w(\bar{Y})\}$. Note that $X=\{\widehat{v}\}$ where $\widehat{v}$ is the first vertex of $\widehat{G}$ obtained by the contraction of one or more edges such that $\widehat{w}(\widehat{v}) \geq \widehat{w}(\widehat{V}) / 6$. Thus, if $\widehat{v}$ is a result of the contraction of a edge $x y$, then $\widehat{w}(\widehat{v})=\widehat{w}(x)+\widehat{w}(y)<$ $\widehat{w}(\widehat{V}) / 6+\widehat{w}(\widehat{V}) / 6=\widehat{w}(\widehat{V}) / 3=w(V) / 3$.

Since $G^{\prime}=G-X$, we conclude that each vertex in $G^{\prime}$ has weight less than $w(V) / 6$. As $w\left(V^{\prime}\right)=$ $w(V)-w(X)>(2 / 3) w(V)$, it follows that each vertex of $G^{\prime}$ has weight less than $w\left(V^{\prime}\right) / 4$. Thus, by Corollary 4 , the connected 2-partition $(Y, \bar{Y})$ of the graph $G^{\prime}$ returned by the algorithm BalBicon $_{2}$ has measure $m \geq w\left(V^{\prime}\right) / 3$. Hence, $m \geq w\left(V^{\prime}\right) / 3>(2 / 9) w(V)$.

Putting together the two cases (a) and (b), we conclude that $m \geq w(V) / 6$; and therefore, opt $(I) \leq 2 m$. This concludes the proof of the theorem.

Corollary 5 Let $I$ be an instance of $\mathrm{BCP}_{3}$ that consists of a 3-connected w-weighted graph $G=(V, E)$. Let $v_{1}=\arg \max \{w(v) \mid v \in V\}$. If $w\left(v_{1}\right)<w(V) / 3$ then the algorithm $\mathcal{A}_{3}$ applied to I returns in polynomial time a connected 3-partition with measure $m$ such that $m \geq w(V) / 6$.

\section{A general framework for connected $q$-partition}

The ideas discussed in Section 4 can be used to obtain a general framework for $\mathrm{BCP}_{q}$ on $q$-connected graphs for $q \geq 4$. We shall discuss later under which conditions we have a 2-approximation for $\mathrm{BCP}_{4}$.

Algorithm $\mathcal{A}_{q}$ Input: A $q$-connected graph $G=(V, E)$ and $w: V \rightarrow Z_{+}$.

Output: A connected $q$-partition of $G(q \geq 4)$.

1. Let $v_{1}=\arg \max \{w(v) \mid v \in V\}$.

2. $V_{1}=\left\{v_{1}\right\}$.

3. If $w\left(V_{1}\right) \geq(1 / q) w(V)$ then

3.1. Let $G^{\prime}=G-V_{1}$ and $w^{\prime}$ the restriction of $w$ to the vertices of $G^{\prime}$.

3.2. $\left(V_{2}, \ldots, V_{q}\right)=\mathcal{A}_{q-1}\left(G^{\prime}, w^{\prime}\right)$.

3.3. return $\left(V_{1}, V_{2}, \ldots, V_{q}\right)$ and halt.

4. If $w\left(V_{1}\right)<\frac{1}{2 q} w(V)$ then

4.1. $(\widehat{G}, \widehat{w})=(G, w) ; \widehat{V}=V$.

4.2. while there is no $\widehat{v} \in \widehat{V}$ such that $\widehat{w}(\widehat{v}) \geq \frac{1}{2 q} \widehat{w}(\widehat{V})$ do $(\widehat{G}, \widehat{w})=$ Contract-edge $(\widehat{G}, \widehat{w})$.

4.3. $V_{1}=\{\widehat{v}\}$, where $\widehat{w}(\widehat{v}) \geq \frac{1}{2 q} \widehat{w}(\widehat{V})$.

5. $G^{\prime}=\widehat{G}-V_{1}, w^{\prime}=\widehat{w}$.

6. $\left(V_{2}, \ldots, V_{q}\right)=\operatorname{Hypo}_{q-1}\left(G^{\prime}, w^{\prime}\right) . / * G^{\prime}$ is 2 -connected $* /$

7. If $V_{1} \neq\left\{v_{1}\right\}$ then recover from $\left(V_{1}, V_{2}, \ldots, V_{q}\right)$ the $q$-partition $\left(V_{1}, V_{2}, \ldots, V_{q}\right)$ of $G$.

8. return $\left(V_{1}, V_{2}, \ldots, V_{q}\right)$. 
At step 6, $\mathrm{Hypo}_{q-1}$ is any algorithm that finds a connected $(q-1)$-partition on a 2-connected graph. If the input graph has high connectivity then the graph $G^{\prime}$ obtained at step 5 is possibly 3 -connected. We know that the graph $\widehat{G}$ obtained at the end of step 4 is 3-connected; and therefore $G^{\prime}$ is at least 2 -connected.

If we had an algorithm $\mathrm{Hypo}_{q-1}$ that finds for a 2-connected graph a connected $(q-1)$-partition with measure at least $w(V) / \alpha$, we could guarantee that $\mathcal{A}_{q}$ has approximation ratio $\max \{2, \alpha /(q-1)\}$.

When $q=4$ we can use the algorithm $\mathcal{A}_{3}$ at step 3.2 and guarantee that the solution returned at step 3.3 is a 2 -approximation. If $w(v) \geq w(V) / 8$ for some vertex $v$ then step 4 is not executed and the 3-partition returned at step 6 - obtained using algorithm $\mathcal{A}_{3}$ - has measure at least $w\left(V^{\prime}\right) / 6$, by Corollary 5 Thus, the solution returned at step 8 has measure at least $w(V) / 8$. This follows from the fact that $w\left(v_{1}\right)<w(V) / 4$ and therefore $w\left(V^{\prime}\right)>(3 / 4) w(V)$. Hence, we have the following result.

Theorem 10 Let I be an instance of $\mathrm{BCP}_{4}$ that consists of a w-weighted 4-connected graph $G=(V, E)$ such that $w(v) \geq \frac{1}{8} w(V)$ for some vertex $v$ in $V$. Then the algorithm $\mathcal{A}_{4}$ applied to $I$ and using $\mathcal{A}_{3}$ at step 6, returns in polynomial time a connected 4-partition with measure $m$ such that $\operatorname{opt}(I) \leq 2 \mathrm{~m}$.

We close this section observing that to use the algorithm $\mathcal{A}_{q}$ for $q \geq 5$, we need an algorithm to obtain a connected $(q-1)$-partition of 2-connected graphs with good guarantee.

\section{Concluding remarks}

We have shown some hardness results for the problem $\mathrm{BCP}_{q}$ and some inapproximability results for $\mathrm{BCP}_{2}$ and $\mathrm{BCP}$. We have also shown 2-approximation algorithms for $\mathrm{BCP}_{3}$ and $\mathrm{BCP}_{4}$ on 3-connected and 4-connected graphs, respectively. We have shown that BCP does not admit a PTAS, but it remains open whether the same holds for $\mathrm{BCP}_{2}$. It would be very interesting to show either the existence or nonexistence of a PTAS for the problem $\mathrm{BCP}_{2}$. Other approximation results for $q>2$ would also be of interest.

The problem $\mathrm{BCP}_{q}$ is related to another problem called Min-max q-Partition Problem, in which the objective is to minimize the 'heaviest' class. We note that these problems are equivalent only when $q=2$. 


\section{References}

[1] P. Alimonti and T. Calamoneri. On the complexity of the max balance problem. In Argentinian Workshop on Theoretical Computer Science (WAIT'99), pages 133 - 138, 1999.

[2] E. Aparo and B. Simeone. Un algoritmo di equipartizione e il suo impiego in un problema de contrasto ottico. Ricerca Operativa, 3:31-42, 1973.

[3] R. Becker, I. Lari, M. Lucertini, and B. Simeone. Max-min partitioning of grid graphs into connected components. Networks, 32:115-125, 1998.

[4] R. Becker, I. Lari, M. Lucertini, and B. Simeone. A polynomial-time algorithm for max-min partitioning of ladders. Theory Comput. Syst., 34(4):353-374, 2001.

[5] R. I. Becker and Y. Perl. Shifting algorithms for tree partitioning with general weighting functions. $J$. Algorithms, 4(2):101-120, 1983.

[6] P. M. Camerini, G. Galbiati, and F. Maffioli. On the complexity of finding multiconstrained spanning trees. Discrete Appl. Math., 5(1):39-50, 1983.

[7] J. Chlebíková. Approximating the maximally balanced connected partition problem in graphs. Inform. Process. Lett., 60(5):225-230, 1996.

[8] M. E. Dyer and A. M. Frieze. On the complexity of partitioning graphs into connected subgraphs. Discrete Appl. Math., 10(2):139-153, 1985.

[9] G. Galbiati, F. Maffioli, and A. Morzenti. On the approximability of some maximum spanning tree problems. volume 911 of Lecture Notes in Computer Science, pages 300-310, 1995.

[10] G. Galbiati, A. Morzenti, and F. Maffioli. On the approximability of some maximum spanning tree problems. Theoret. Comput. Sci., 181(1):107-118, 1997. Latin American Theoretical INformatics (Valparaíso, 1995).

[11] M. R. Garey and D. S. Johnson. Computers and intractability: a guide to the theory of NPcompleteness. W. H. Freeman and Co., San Francisco, Calif., 1979. A Series of Books in the Mathematical Sciences.

[12] E. Győri. On division of graphs to connected subgraphs. In Combinatorics (Proc. Fifth Hungarian Colloq., Keszthely, 1976), Vol. I, volume 18 of Colloq. Math. Soc. János Bolyai, pages 485-494. North-Holland, Amsterdam, 1978.

[13] L. Lovász. A homology theory for spanning trees of a graph. Acta Math. Acad. Sci. Hungar., 30 (3-4):241-251, 1977.

[14] M. Lucertini, Y. Perl, and B. Simeone. Image enhancement by path partitioning. In V. Cantoni and S. Levialdi, editors, Recent Issues in Image Analysis, Lecture Notes in Computer Science. Springer, 1989.

[15] M. Lucertini, Y. Perl, and B. Simeone. Most uniform path partitioning and its use in image processing. Discrete Appl. Math., 42(2-3):227-256, 1993. 
[16] J. Ma and S. H. Ma. An $O\left(k^{2} n^{2}\right)$ algorithm to find a $k$-partition in a $k$-connected graph. J. Comput. Sci. Tech., 9(1):86-91, 1994.

[17] M. Maravalle, B. Simeone, and R. Naldini. Clustering on trees. Comput. Statist. Data Anal., 24(2): 217-234, 1997.

[18] S. Nakano, M. S. Rahman, and T. Nishizeki. A linear-time algorithm for four-partitioning fourconnected planar graphs. Inform. Process. Lett., 62(6):315-322, 1997.

[19] C. H. Papadimitriou and M. Yannakakis. The complexity of restricted spanning tree problems. $J$. Assoc. Comput. Mach., 29(2):285-309, 1982.

[20] Y. Perl and S. R. Schach. Max-min tree partitioning. J. Assoc. Comput. Mach., 28(1):5-15, 1981.

[21] L. R. B. Salgado. Algoritmos de Aproximação para Partições Conexas em Grafos. PhD thesis, Universidade de São Paulo, 2004.

[22] L. R. B. Salgado and Y. Wakabayashi. Approximation results on balanced connected partitions of graphs. In Latin-American Conference on Combinatorics, Graphs and Applications, volume 18 of Electron. Notes Discrete Math., pages 207-212 (electronic). Elsevier, Amsterdam, 2004.

[23] H. Suzuki, N. Takahashi, and T. Nishizeki. A linear algorithm for bipartition of biconnected graphs. Inform. Process. Lett., 33(5):227-231, 1990.

[24] H. Suzuki, N. Takahashi, T. Nishizeki, H. Miyano, and S. Ueno. An algorithm for tripartitioning 3-connected graphs. Journal of Information Processing Society of Japan, 31(5):584-592, 1990. 